\title{
Protective effects of propofol on experimental neonatal acute lung injury
}

\author{
XIONGWU YU and CHUANFENG LI

\begin{abstract}
Department of Pediatric Surgery, Maternal and Child Health Care Hospital of Qujing, Qujing, Yunnan 655000, P.R. China
\end{abstract}
Received May 25, 2018; Accepted December 17, 2018

DOI: $10.3892 / \mathrm{mmr} .2019 .10113$

\begin{abstract}
The present study aimed to investigate the effects of propofol on neonatal acute lung injury (ALI) in a rat model and to examine the molecular mechanisms underlying propofol function. A rat model of ALI was established by intraperitoneal injection of lipopolysaccharides (LPS). The neonatal rats were treated with various concentrations of propofol and a lung injury score was assessed. The protein expression levels of pro-inflammatory cytokines was detected using ELISA. In the present study, oxidative stress was determined by measuring the level of malondialdehyde (MDA) and the activity of superoxide dismutase (SOD) in lung tissues. Reverse transcription quantitative-polymerase chain reaction and western blot analysis were used to examine the mRNA and protein expression levels of the factors downstream to LPS signaling pathway. Treatment with propofol significantly alleviated LPS-induced lung injury in neonatal rats as suggested by the decreased lung injury score, increased partial pressure of oxygen and decreased lung wet-dry weight ratio. LPS promoted the upregulation of tumor necrosis factor $\alpha$ (TNF- $\alpha$ ), interleukin (IL)- 6 and IL-1 $\beta$ in lung tissues and bronchoalveolar lavage fluid from neonatal rats exhibiting ALI. Notably, treatment with propofol decreased the expression levels of these factors. Additionally, LPS caused an increase in the levels of MDA, and a decrease in SOD activity, and treatment with propofol suppressed these effects in a dose-dependent manner. Furthermore, LPS induced the upregulation of phosphorylated (p-)p38, nuclear factor $\kappa$-light-chain-enhancer of activated $B$ cells $(\mathrm{NF}-\kappa \mathrm{B}), \mathrm{p}-\mathrm{p} 65$, NLR family pyrin domain containing 3 (NLRP3), apoptosis-associated speck-like protein containing CARD and caspase-1 in lung tissues of neonatal rats, and treatment with propofol was able to downregulate these factors in a dose-dependent manner. Propofol alleviated lung
\end{abstract}

Correspondence to: Dr Chuanfeng Li, Department of Pediatric Surgery, Maternal and Child Health Care Hospital of Qujing, 371 Liaokuo South Road, Qujing, Yunnan 655000, P.R. China E-mail: licf201717@163.com

Key words: neonatal acute lung injury, inflammation, oxidative stress, p38 mitogen-activated protein kinase/nuclear factor $\kappa$-light-chain-enhancer of activated B cells pathway, NLR family pyrin domain containing 3 inflammasome injury in neonatal rats with LPS-induced ALI by preventing inflammation and oxidative stress via the regulation of the activity of the p38 mitogen-activated protein kinase/NF- $\kappa \mathrm{B}$ signaling pathway and the expression levels of the NLRP3 inflammasome.

\section{Introduction}

Acute lung injury (ALI) is a disorder associated with pulmonary inflammation that may lead to increased permeability syndrome (1). Various factors that may not be involved in the respiratory or in the cardiovascular system are involved in the pathogenesis of ALI (2). ALI is characterized by pulmonary edema and increased lung permeability caused by diffuse alveolar capillary injury that may lead to acute and progressive hypoxic respiratory failure (3). ALI is caused by the extensive destruction of pulmonary vascular and alveolar epithelial cells following severe inflammation (4). ALI exhibits significant morbidity and mortality rates with poor outcomes in patients of all ages (5). In addition, ALI is one of the most common pediatric diseases and one of the most frequent causes of neonatal mortality (6). The estimated mortality rate of ALI is $38.6-65 \%$, and previous studies demonstrated that the mortality rate varied by geographical region $(7,8)$. Although the use of lung protective strategies including ventilation, prolonged prone position, neuromuscular blockade, treatment with inhalable vasodilators and nonpulmonary treatments have improved the outcomes of ALI, the treatment of ALI remains challenging $(9,10)$. Therefore, there is an urgent requirement to identify novel effective therapies against ALI.

Lipopolysaccharides (LPS) are bacterial bioactive components involved in various pathological conditions and are able to promote the inflammatory cascade (11-13). Previous studies used LPS for the establishment of animal models of ALI (11-13). Therefore, in the present study, a rat model of ALI was established by intraperitoneal injection of LPS.

Propofol is used to induce or maintain anesthesia (14). Nevertheless, accumulating evidence demonstrated that propofol serve multiple functions, including anticancer, antioxidant, neuroprotective and anti-inflammatory activities (15-20). The effect of propofol in lung injury has been previously investigated; Yuan et al (21) observed that Propofol was able to relieve ALI induced by liver transplantation by inhibiting gap junction protein $\alpha 1$. Liu et al (22) suggested that propofol increased the effect of sevoflurane in relieving 
LPS-induced ALI in mice. A recent study demonstrated that treatment with propofol significantly increased the expression levels of heme oxygenase 1 in lung tissues and inhibited the lung morphological alterations caused by ALI in rats (23). Additionally, the p38 mitogen-activated protein kinase (MAPK)/nuclear factor $\kappa$-light-chain-enhancer of activated B cells $(\mathrm{NF}-\kappa \mathrm{B})$ signaling pathway and the NLR family pyrin domain containing 3 (NLRP3) inflammasome, which serve roles in the regulation of inflammation, were identified to be regulated by propofol $(20,24)$. A number of previous studies demonstrated that the p38 MAPK/NF- $\kappa$ B pathway was activated during ALI $(25,26)$. Furthermore, previous studies suggested that the components of the NLRP3 inflammasome including NLRP3, apoptosis-associated speck-like protein containing CARD (ASC) and caspase-1, serve critical roles in ALI development and progression by regulating the inflammatory response $(27,28)$.

The mechanism underlying propofol effect on lung injury remains unclear. Specifically, to the best of the authors' knowledge, the effect of propofol on neonatal ALI has not been examined. The present study aimed to investigate the role of propofol on neonatal ALI and the molecular mechanism underlying its effects.

\section{Materials and methods}

Establishment of the ALI model. A total of 30 male newborn Sprague-Dawley rats (age, 3-8 days; weight, 8-14 g) were obtained from the Animal Experimental Center of Zhejiang University (Hangzhou, China). All experimental procedures were performed according to the Recommended Guidelines for the Care and Use of Laboratory Animals issued by The Chinese Council on Animal Research (29). The present study was approved by The Animal Ethics Committee of The Maternal and Child Health Care Hospital of Qujing (Qujing, China). Rat pups were kept in polypropylene cages with nursing mothers. The nursing mothers were fed ad libitum Rats were housed at $25 \pm 5^{\circ} \mathrm{C}$, with $50 \%$ humidity and $12 \mathrm{~h}$ dark/light cycle conditions.

Toinduce ALI, rats were injected intraperitoneally with LPS ( 3 mg/kg; Sigma-Aldrich; Merck KGaA, Darmstadt, Germany) as previously described (13) and successful establishment of the model was confirmed by lung injury scores. Then, $1 \mathrm{~h}$ after treatment with LPS, rats were additionally treated by intraperitoneal injection of various concentrations of propofol (Corden Pharma Caponago S.P.A., Milan, Italy) (1, 5 and $10 \mathrm{mg} / \mathrm{kg})$ once a day for 7 consecutive days. Propofol working concentrations were selected according to a previous study (30). Rats were randomly divided into six groups ( $n=5$ per group): i) Control group, ii) LPS group ( $3 \mathrm{mg} / \mathrm{kg})$, iii) LPS + propofol (1 $\mathrm{mg} / \mathrm{kg})$ group, iv) LPS + propofol $(5 \mathrm{mg} / \mathrm{kg})$ group and v) LPS + propofol (10 mg/kg) group.

Lung injury score assessment. To perform the assessment of the lung injury score, lung tissues were collected from the control and the three experimental groups. Lung tissues were fixed with $10 \%$ neutral phosphate-buffered formalin at $4^{\circ} \mathrm{C}$ for $24 \mathrm{~h}$, embedded in paraffin and sectioned $(4 \mu \mathrm{m})$. Subsequently, hematoxylin and eosin staining was conducted using standard protocols. The pathological alterations in the morphology of lung tissues were observed under a light microscope (magnification, x200). The lung injury score was assessed to quantify the lung injury. Lung injury scores were calculated by summing the degree of cell infiltration and the severity score of tissue damage assessed from the lung sections (31).

Lung edema assessment. Rats were anesthetized with 3\% isoflurane. Blood samples $(200 \mu \mathrm{l})$ were collected from abdominal aorta, and an automatic blood gas analyzer (Cobas B123; Roche Diagnostics, Basel, Switzerland) was used to measure the partial pressure of oxygen $\left(\mathrm{PaO}_{2}\right)$. The rats were sacrificed and the right lungs were collected from the control and the three experimental groups. Subsequently, the wet weights (W) of the right lungs were measured. Subsequently, following a 48-h incubation at $70^{\circ} \mathrm{C}$, the dry weights (D) of the right lungs were measured and the wet-dry weight ratio (W/D) was calculated.

Measurement of inflammatory factors and oxidative stress-associated factors. The bronchoalveolar lavage fluid (BALF) was obtained by intratracheal instillation of sterile PBS, repeated three times. The BALF supernatant was collected by centrifugation $(800 \mathrm{x} \mathrm{g})$ at $4^{\circ} \mathrm{C}$ for $10 \mathrm{~min}$. ELISA kits (Wuhan Boster Biological Technology, Ltd., Wuhan, China) were used to detect the levels in BALF of the following pro-inflammatory cytokines: Tumor necrosis factor $\alpha$ (TNF- $\alpha$; cat. no. EK0526), interleukin (IL)-1 $\beta$ (cat. no. EK0393) and IL-6 (cat. no. EK0412). The level of malondialdehyde (MDA) and the activity of superoxide dismutase (SOD) in lung tissues were determined using commercially available kits (cat. nos. A003-1 and A001-3; Nanjing Jiancheng Bioengineering Institute, Nanjing, China) according to the manufacturer's protocol.

Reverse transcription quantitative-polymerase chain reaction $(R T-q P C R)$. Total RNA was isolated from lung tissues by using TRIzol ${ }^{\circledR}$ reagent (Invitrogen; Thermo Fisher Scientific, Inc., Waltham, MA, USA) according to the manufacturer's protocol. An equal amount of RNA from various groups was reversely transcribed into cDNA using PrimeScript ${ }^{\mathrm{TM}}$ RT reagent kit (Takara Bio Inc., Otsu, Japan) according to the manufacturer's protocol. qPCR was performed using SYBR ${ }^{\circledR}$ Premix Ex $\mathrm{Taq}^{\mathrm{TM}}$ II (Takara Bio Inc.). Thermocycling conditions were: $95^{\circ} \mathrm{C}$ for $5 \mathrm{~min}, 40$ cycles at $95^{\circ} \mathrm{C}$ for $30 \mathrm{sec}, 55^{\circ} \mathrm{C}$ for $30 \mathrm{sec}$, $72^{\circ} \mathrm{C}$ for $30 \mathrm{sec}$ and then $72^{\circ} \mathrm{C}$ for $10 \mathrm{~min}$. GAPDH was used as endogenous control. Primer sequences are presented in Table I. Relative mRNA expression levels were normalized to GAPDH using the $2^{-\Delta \Delta \mathrm{Cq}}$ method (32).

Western blot assay. Total proteins from whole tissue lysates were extracted using radioimmunoprecipitation assay buffer (cat. no. P0013B; Beyotime Institute of Biotechnology, Nanjing, China), and a bicinchoninic acid assay kit was used to quantify protein concentration. A total of $25 \mu \mathrm{g}$ protein was loaded per lane, in a volume of $20 \mu \mathrm{l}$. Proteins were separated by $12 \%$ SDS-PAGE, and subsequently transferred onto polyvinylidene fluoride membranes (EMD Millipore, Billerica, MA, USA), and blocked with 5\% skim milk for $1 \mathrm{~h}$ at room temperature. The membranes were incubated at $4^{\circ} \mathrm{C}$ overnight with one of the following primary antibodies: TNF- $\alpha$ (cat no. ab13597; 1:1,000; Abcam, Cambridge, MA, USA), IL-1 $\beta$ (cat no. ab200478; 1:1,000; Abcam), IL-6 (cat no. ab9324; 
Table I. Primer sequences used for the reverse transcription quantitative-polymerase chain reaction.

\begin{tabular}{ll}
\hline Gene & \multicolumn{1}{c}{ Primer sequence $\left(5^{\prime} \rightarrow 3^{\prime}\right)$} \\
\hline TNF- $\alpha$ & F: CCTCTTCTCATTCCTGCTC \\
& R: CTTCTCCTCCTTG TTGGG \\
IL-1 $\beta$ & F: TGTGAAATGCCACCTTTTGA \\
& R: TGAGTGATACTGCCTGCCTG \\
IL-6 & F: CCGGAGAGGAGACTTCACAG \\
& R: CAGAATTGCCATTGCACA \\
NLRP3 & F: GATCTTCGCTGCGATCAACAG \\
& R: CGTGCATTATCTGAACCCCAC \\
ASC & F: GCAATGTGCTGACTGAAGGA \\
& R: TGTTCCAGGTCTGTCACCAA \\
Faspase-1 & R: GCACAAGACCTCTGACAGCA \\
& F: CTTTGGTATCATCTTGGTATC \\
GAPDH & R:GTAGAGGCAGGGATGATGTTCT
\end{tabular}

ASC, apoptosis-associated speck-like protein containing CARD; F, forward; IL, interleukin; NLRP3, NLR family pyrin domain containing 3 ; R, reverse; TNF- $\alpha$, tumor necrosis factor- $\alpha$.

1:1,000; Abcam), phosphorylated (p-)p38 (cat no. ab45381; 1:1,000; Abcam), p38 (cat no. ab31828; 1:1,000; Abcam), p-p65 (cat no. ab86299; 1:1,000; Abcam), p65 (cat no. ab16502; 1:1,000; Abcam), NLRP3 (cat no. ab214185; 1:1,000; Abcam), ASC (at no. sc-514414; Santa Cruz Biotechnology, Inc., Dallas, TX, USA), caspase-1 (cat no. ab1872; 1:1,000; Abcam) and $\beta$-actin (cat no. 4970; 1:1,000; Cell Signaling Technology, Inc., Danvers, MA, USA). Subsequently, the membranes were incubated with the anti-rabbit immunoglobulin $\mathrm{G}$ horseradish peroxidase-labeled secondary antibody (cat no. 7074; 1:2,000; Cell Signaling Technology, Inc.) at room temperature for $2 \mathrm{~h}$. Enhanced chemiluminescence reagents (EMD Millipore) were used to visualize the protein bands. The intensity of p-p38 and p-p65 was analyzed using Gel-Pro-Analyzer software version 4.0 (Media Cybernetics, Inc., Rockville, MD, USA).

Statistical analysis. Data are presented as the mean \pm standard deviation. SPSS statistical software (version 16.0; SPSS, Inc., Chicago, IL, USA) was used to conduct statistical analyses. One-way analysis of variance followed by Student-Newman-Keuls post hoc test was performed to determine differences between groups. $\mathrm{P}<0.05$ was considered to indicate a statistically significant difference.

\section{Results}

Propofol relieves the pulmonary edema induced by LPS in neonatal rats. Treatment with propofol decreased the lung injury score in a dose-dependent manner in ALI Model rats, which was significantly increased upon treatment with LPS (Fig. 1A). Furthermore, treatment with LPS significantly decreased the $\mathrm{PaO}_{2}$ and increased the W/D ratio in neonatal rats (Fig. $1 \mathrm{~B}$ and $\mathrm{C}$, respectively). However, treatment with propofol significantly reversed the effects detected in
LPS-associated ALI in neonatal rats in a dose-dependent manner (Fig. 1B and C, respectively).

Propofol prevents the inflammatory response induced by LPS in neonatal rats. Inflammation serves an important role in the initiation and maintenance of ALI (33), and the principal feature of ALI-associated inflammation is the upregulation of pro-inflammatory factors, including TNF- $\alpha$, IL-1 $\beta$ and IL-6 (34-36). The present study investigated the effects of propofol on the protein expression levels of TNF- $\alpha$, IL- 6 and IL- $1 \beta$ in the BALF of neonatal rats by ELISA (Fig. 2). The results demonstrated that LPS significantly increased the protein expression levels of TNF- $\alpha$, IL- 6 and IL- $1 \beta$ in the BALF of neonatal rats, whereas, treatment with propofol decreased their concentrations in a dose-dependent manner (Fig. 2). Additionally, the protein and mRNA expression levels of TNF- $\alpha$, IL- 6 and IL-1 $\beta$ were measured by western blot analysis and RT-qPCR, respectively. The protein and mRNA expression levels of TNF- $\alpha$, IL- 6 and IL-1 $\beta$ in lung tissues of neonatal rats were increased by treatment with LPS, and propofol decreased their expression levels in a dose-dependent manner (Fig. 3).

Propofol prevents the oxidative stress induced by LPS in neonatal rats. The effects of propofol on the oxidative stress induced by ALI were examined. Compared with the control group, treatment with LPS significantly decreased the activity of SOD and increased the level of MDA in lung tissues (Fig. 4A and B, respectively). The activity of SOD was significantly increased and the level of MDA was significantly decreased following treatment with propofol in neonatal rats with ALI, compared with the Model ALI rats (Fig. 4). These results suggested that propofol suppressed the oxidative stress detected in neonatal rats with ALI.

Propofol prevents the activation of the p38 MAPK/NF- $\mathrm{BB}$ signaling pathway induced by LPS in neonatal rats. The expression level of pro-inflammatory cytokines is regulated at the transcriptional level by the MAPK and NF- $\mathrm{B}$ signaling pathways, and previous studies demonstrated that the p38 MAPK/NF- $\kappa$ B pathway is active during ALI $(25,26)$. In the present study, to investigate the molecular mechanism underlying the effects of propofol on ALI, the p38 MAPK/NF- $\kappa$ B signaling pathway was investigated. Treatment with LPS significantly increased the protein expression levels of p-p38 and p-p65 in the lung tissues of neonatal rats, which suggested that the activity of the $\mathrm{p} 38 \mathrm{MAPK} / \mathrm{NF}-\kappa \mathrm{B}$ signaling pathway was increased. Furthermore, treatment with propofol was identified to decrease the protein expression level of p-p38 and p-p65 in neonatal ALI model rats in a dose-dependent manner (Fig. 5).

Propofol prevents the activation of the NLRP3 inflammasome induced by LPS in neonatal rats. The NLRP3 inflammasome serves an important role in regulating the inflammatory response and oxidative stress (37). Numerous previous studies demonstrated that the NLRP3 inflammasome, consisting of NLRP3, ASC and caspase-1, was activated during ALI and it may serve critical roles in ALI development by promoting inflammation $(27,28)$. Therefore, the components of the NLRP3 inflammasome were investigated in the present study. 

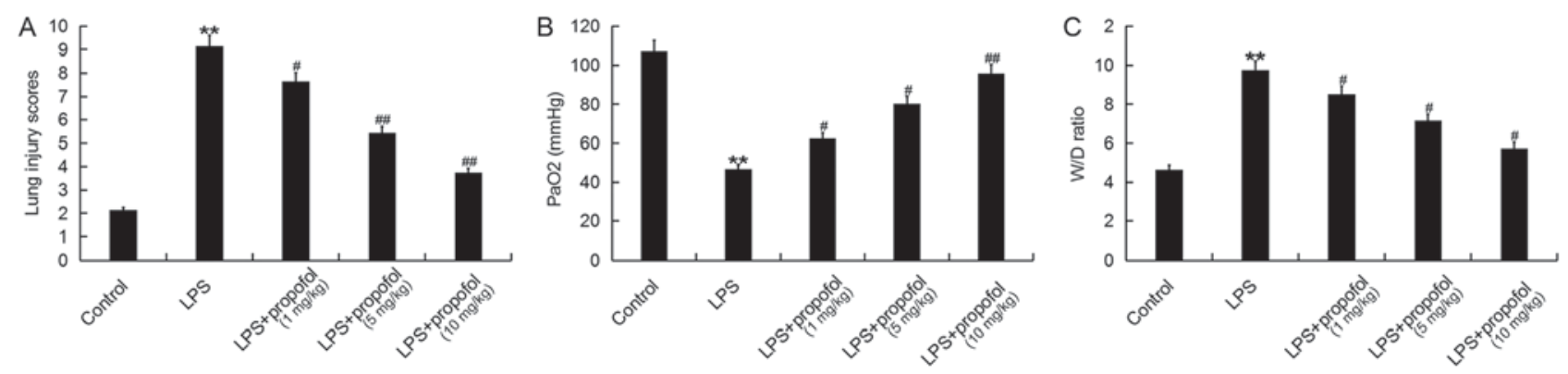

Figure 1. Propofol relieves the pulmonary edema induced by LPS in neonatal rats. (A) Lung injury scores in neonatal rats with LPS-induced acute lung injury treated with various concentration of propofol. (B) $\mathrm{PaO}_{2}$ in arterial blood samples collected from neonatal rats treated with LPS and propofol. (C) Lung W/D in neonatal rats treated with LPS and propofol. ${ }^{* *} \mathrm{P}<0.01$ vs. control group; ${ }^{\#} \mathrm{P}<0.05,{ }^{\# \#} \mathrm{P}<0.01$ vs. LPS group. LPS, lipopolysaccharides; $\mathrm{PaO}_{2}$, partial pressure of oxygen; W/D, wet-dry weight ratio.
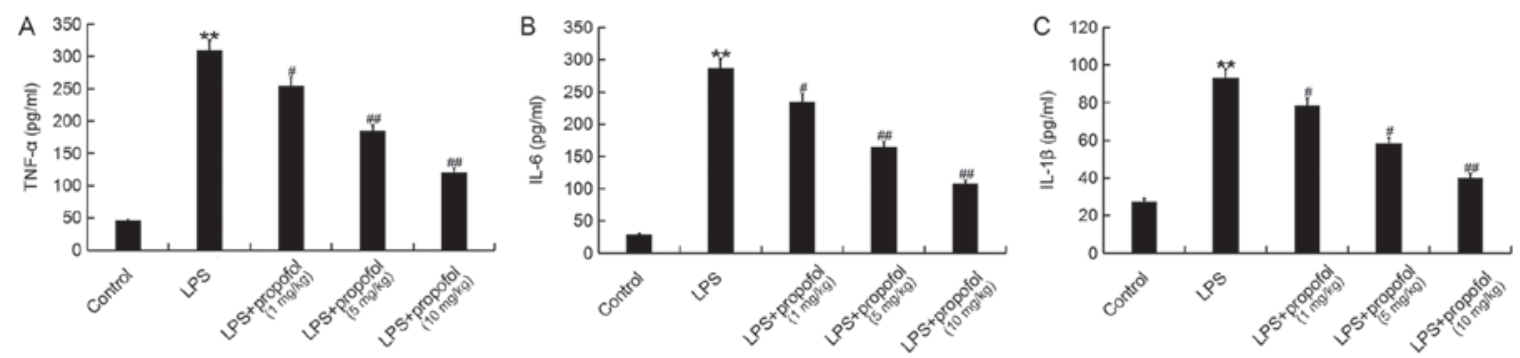

Figure 2. Propofol prevents the inflammatory response induced by LPS in neonatal rats. Protein expression level of (A) TNF- $\alpha$, (B) IL-6 and (C) IL-1 $\beta$ in bronchoalveolar lavage fluid collected from neonatal rats treated with LPS and propofol. ${ }^{* * *} \mathrm{P}<0.01$ vs. control group; ${ }^{~} \mathrm{P}<0.05$, ${ }^{\# \#} \mathrm{P}<0.01$ vs. LPS group. LPS, lipopolysaccharides; TNF- $\alpha$, tumor necrosis factor $\alpha$; IL, interleukin.
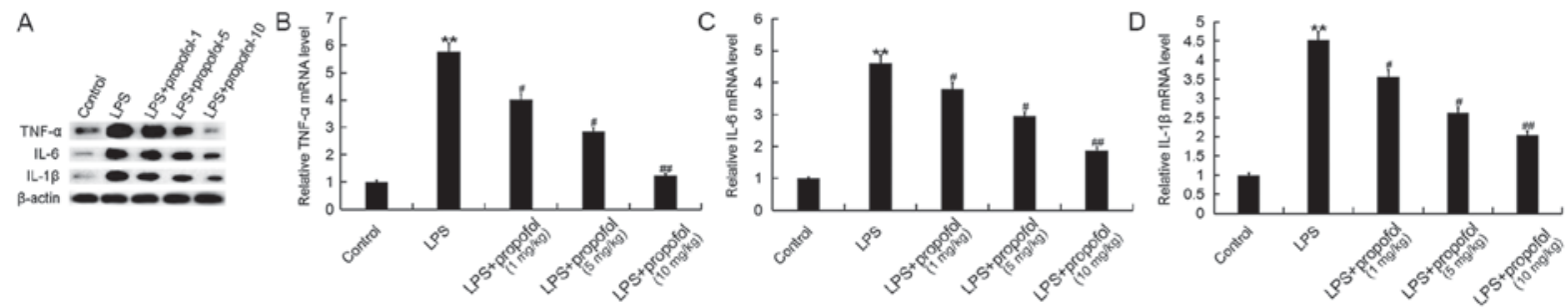

Figure 3. Propofol decreases the expression levels of inflammatory cytokines promoted by LPS in neonatal rats. (A) Protein expression level of TNF- $\alpha$, IL-6 and IL-1 $\beta$ in lung tissues collected from neonatal rats treated with LPS and propofol. mRNA expression level of (B) TNF- $\alpha$, (C) IL- 6 and (D) IL-1 $\beta$ in lung tissues collected from neonatal rats treated with LPS and propofol. ${ }^{* *} \mathrm{P}<0.01$ vs. control group; ${ }^{*} \mathrm{P}<0.05,{ }^{\# \#} \mathrm{P}<0.01$ vs. LPS group. LPS, lipopolysaccharides; TNF- $\alpha$, tumor necrosis factor $\alpha$; IL, interleukin.

The protein and mRNA expression levels of NLRP3, ASC and caspase-1 in lung tissues were significantly increased following treatment with LPS, and these effects were reversed by the treatment with propofol in a dose-dependent manner (Fig. 6).

\section{Discussion}

In the present study, treatment with propofol was identified to relieve LPS-induced pulmonary edema and to inhibit LPS-mediated inflammatory response and oxidative stress in neonatal rats. In addition, the present findings suggested that treatment with propofol inhibited the activation of the p38-MAPK/NF- $\kappa$ B pathway and NLRP3 inflammasome. These results suggested that treatment with propofol may alleviate ALI induced by LPS administration.

Pediatric ALI is one of the most common causes of infant mortality, as newborns are more vulnerable than adults $(38,39)$. Unfortunately, diagnosis of ALI remains challenging and no treatments are available, despite the high morbidity and mortality rates of ALI. Therefore, there is an urgent requirement to identify novel efficient strategies to treat ALI. Propofol is frequently used as an anesthetic; however, it possesses a variety of activities, including anti-inflammatory activity (15-20). Although previous studies observed the protective effects of propofol in ALI (21-23), its mechanism of action requires further investigation, and the role of propofol in neonatal ALI has not been previously examined, to the best of the authors' knowledge. The present study aimed to investigate the effects of propofol on neonatal ALI and to investigate its mechanism of action.

As one of the early symptoms of multiple organ failure, the onset of ALI is associated with increased circulation levels of endotoxin or LPS (39). LPS is considered the most important antigen for the development of ALI (40). Furthermore, animal 

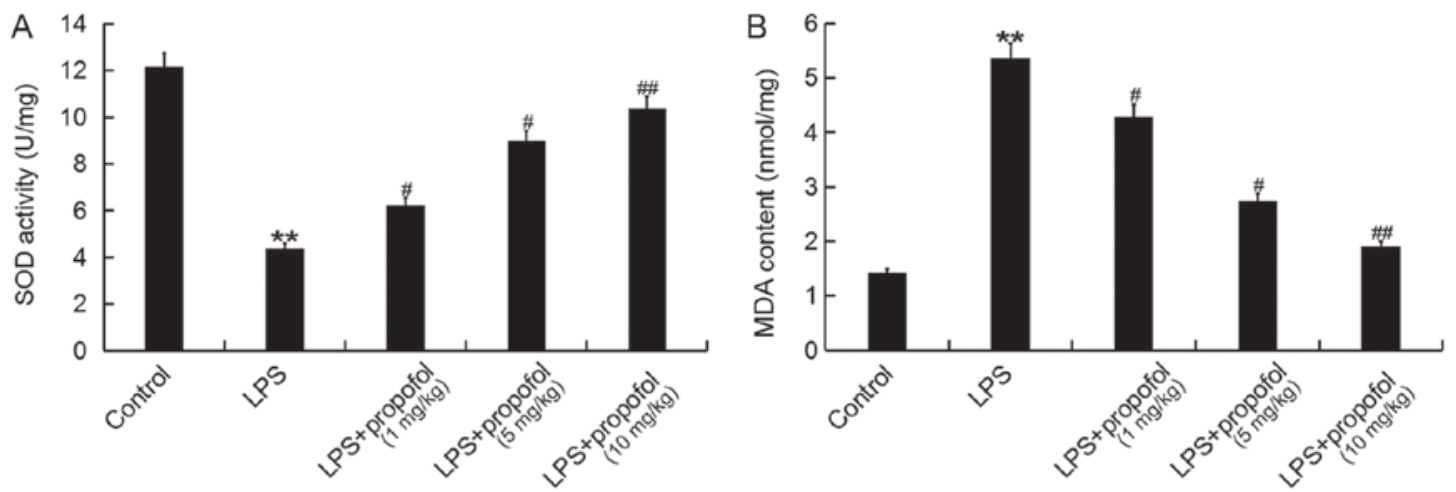

Figure 4. Propofol prevents the oxidative stress induced by LPS in neonatal rats. (A) SOD activity in lung tissues collected from neonatal rats treated with LPS and propofol. (B) Concentration of MDA in lung tissues collected from neonatal rats treated with LPS and propofol. ${ }^{* * *} \mathrm{P}<0.01 \mathrm{vs}$. control group; ${ }^{~} \mathrm{P}<0.05$, ${ }^{\# \#} \mathrm{P}<0.01$ vs. LPS group. LPS, lipopolysaccharides; SOD, superoxide dismutase; MDA, malondialdehyde.
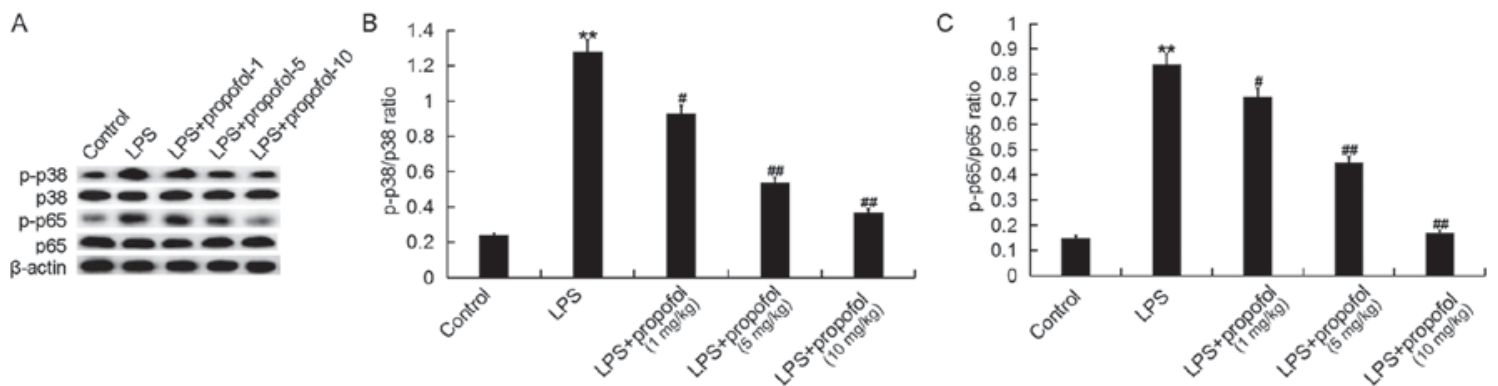

Figure 5. Propofol prevents the activation of the p38 mitogen-activated protein kinase/nuclear factor $\kappa$-light-chain-enhancer of activated B cells signaling pathway induced by LPS in neonatal rats. (A) Protein expression levels of p-p38, p38, p65 and p-p65 in lung tissues collected from neonatal rats treated with LPS and propofol were detected by western blot assay. The ratio of (B) p-p38/p38 and (C) p-p65/p65 in lung tissues collected from neonatal rats treated with LPS and propofol. ${ }^{* *} \mathrm{P}<0.01$ vs. control group; ${ }^{\# P} \mathrm{P}<0.05,{ }^{\# \#} \mathrm{P}<0.01$ vs. LPS group. ASC, apoptosis-associated speck-like protein containing CARD; LPS, lipopolysaccharides; NLRP3, NLR family pyrin domain containing 3; $\mathrm{p}$-, phosphorylated.

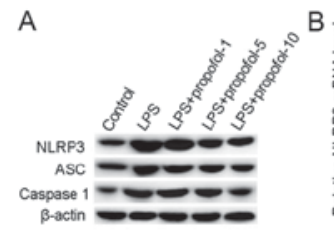

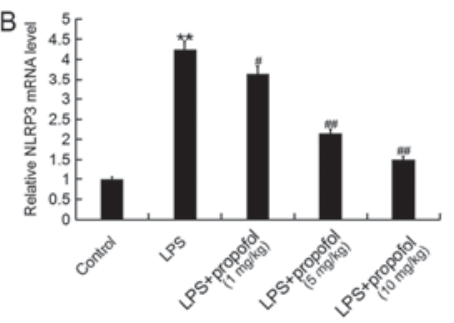
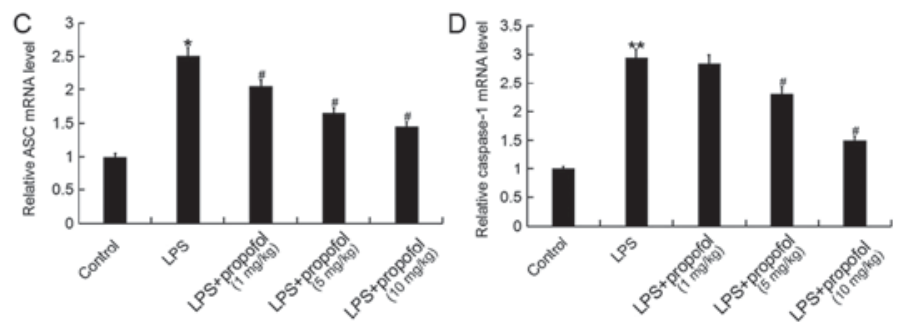

Figure 6. Propofol prevents the activation of the NLRP3 inflammasome induced by LPS in neonatal rats. (A) Protein expression levels of NLRP3, ASC and caspase-1 in lung tissues collected from neonatal rats treated with LPS and propofol were detected by western blot assay. mRNA expression levels of (B) NLRP3, (C) ASC and (D) caspase-1 in lung tissues collected from neonatal rats treated with LPS and propofol were analyzed using reverse transcription quantitative-polymerase chain reaction. ${ }^{*} \mathrm{P}<0.05,{ }^{* *} \mathrm{P}<0.01$ vs. control group; ${ }^{*} \mathrm{P}<0.05,{ }^{\# \prime} \mathrm{P}<0.01$ vs. LPS group. ASC, apoptosis-associated speck-like protein containing CARD; LPS, lipopolysaccharides; NLRP3, NLR family pyrin domain containing 3.

models of LPS-induced ALI are frequently used to investigate ALI (13,41-43). Therefore, in the present study, LPS was used to establish an animal model of ALI in neonatal rats, and various concentrations of propofol were administrated by intraperitoneal injection. In a previous studies, to assess lung edema, the lung W/D ratio of neonatal rats was determined (44), and the level of arterial blood $\mathrm{PaO}_{2}$ was examined to investigate the pulmonary gas exchange function (44). Lung injury scores were calculated in a previous study by summing the degree of cell infiltration and the severity score of tissue damage was assessed from observation of the lung sections (31). In the present study treatment with propofol was able to relieve the pulmonary edema and to alleviate the lung injury induced by LPS in neonatal rats. Accumulating evidence demonstrated that inflammation serves a key role in the initiation and maintenance of ALI (33), and inflammation in animal models of ALI is characterized by an increase in the circulating levels of inflammatory factors including TNF- $\alpha$, IL-1 $\beta$ and IL-6 (34-36).

The effects of propofol on the protein expression levels of certain inflammatory factors were examined in neonatal rats with ALI. The present results suggested that propofol decreased the protein expression levels of TNF- $\alpha$, IL- 6 and IL- $1 \beta$ that were increased by LPS in neonatal rats, which is consistent with a 
previous study (22). Oxidative stress, which has been identified to be involved in LPS-induced ALI $(45,46)$, was also assessed in the present study. Treatment with propofol was identified to significantly suppress oxidative stress in neonatal rats with ALI.

To investigate the molecular mechanism underlying the effects of propofol on neonatal ALI, the present study analyzed the p38 MAPK/NF- $\mathrm{KB}$ pathway and NLRP3 inflammasome, which were previously identified to serve important roles in the regulation of the inflammatory response and to be regulated by propofol $(20,21)$. The present study results suggested that the p38 MAPK/NF- $\mathrm{KB}$ pathway and NLRP3 inflammasome were activated by the treatment with LPS in neonatal rats, and treatment with propofol significantly prevented the activation of $\mathrm{p} 38$ MAPK/NF- $\kappa$ B signaling pathway and NLRP3 inflammasome.

However, due to its rapid onset and short elimination half-life, propofol is considered an addictive substance with sedative and relaxing effects, and the recreational abuse of this drug has increased over the past years $(47,48)$. Although the concentration of propofol used in the present study is not sufficient to induce addiction $(48,49)$, propofol is an addictive drug and treatment with propofol requires to be monitored. Therefore, future studies are required to further investigate the side effects of propofol prior to its clinical use.

In summary, the present study suggested that treatment with propofol may relieve LPS-induced pulmonary edema, inhibit LPS-associated inflammatory response and oxidative stress in neonatal rats by repressing the activation of the $\mathrm{p} 38$ MAPK/NF- $\mathrm{KB}$ signaling pathway and the NLRP3 inflammasome. Propofol served a protective role in neonatal ALI induced by LPS, and it may represent a promising therapeutic agent for the treatment of neonatal ALI.

\section{Acknowledgements}

The authors would like to thank Dr Deng Xingmei from The Department of Paediatrics, Maternal and Child Health-Care Hospital for his help.

\section{Funding}

No funding was received.

\section{Availability of data and materials}

The datasets used and/or analyzed during the current study are available from the corresponding author on reasonable request.

\section{Authors' contributions}

$\mathrm{XY}$ was responsible for study design, data access and analysis, interpretation of results and preparation of the manuscript. CL was responsible for interpretation of results and preparation of the manuscript.

\section{Ethics approval and consent to participate}

All experimental procedures were performed according to the Recommended Guidelines for the Care and Use of Laboratory Animals issued by The Chinese Council on Animal Research. The present study was approved by The Animal Ethics
Committee of The Maternal and Child Health-Care Hospital of Qujing (Qujing, China).

\section{Patient consent for publication}

Not applicable.

\section{Competing interests}

The authors declare that they have no competing interests.

\section{References}

1. Rubenfeld GD, Caldwell E, Peabody E, Weaver J, Martin DP, Neff M, Stern EJ and Hudson LD: Incidence and outcomes of acute lung injury. N Engl J Med 353: 1685-1693, 2005.

2. Butt $Y$, Kurdowska A and Allen TC: Acute lung injury: A clinical and molecular review. Arch Pathol Lab Med 140: 345-350, 2016.

3. Cheifetz IML: Year in review 2015: Pediatric ARDS. Respir Care 61: 980-985, 2016.

4. Guo Z, Gu Y, Wang C, Zhang J, Shan S, Gu X, Wang K, Han Y and Ren T: Enforced expression of miR-125b attenuates LPS-induced acute lung injury. Immunol Lett 162: 18-26, 2014.

5. De Luca D, Piastra M, Tosi F, Pulitanò S, Mancino A, Genovese O, Pietrini D and Conti G: Pharmacological therapies for pediatric and neonatal ALI/ARDS: An evidence-based review. Curr Drug Targets 13: 906-916, 2012.

6. Chakraborty M, McGreal EP and Kotecha S: Acute lung injury in preterm newborn infants: Mechanisms and management. Paediatr Respir Rev 11: 162-170, 2010.

7. Fujishima S, Gando S, Daizoh S, Kushimoto S, Ogura H, Mayumi T, Takuma K, Kotani J, Yamashita N, Tsuruta R, et al: Infection site is predictive of outcome in acute lung injury associated with severe sepsis and septic shock. Respirology 21: 898-904, 2016.

8. Quasney MW, López-Fernández YM, Santschi M and Watson RS; Pediatric Acute Lung Injury Consensus Conference Group: The outcomes of children with pediatric acute respiratory distress syndrome: Proceedings from the pediatric acute lung injury consensus conference. Pediatr Crit Care Med 16 (5 Suppl 1): S118-S131, 2015.

9. Pediatric Acute Lung Injury Consensus Conference Group: Pediatric acute respiratory distress syndrome: Consensus recommendations from the pediatric acute lung injury consensus conference. Pediatr Crit Care Med 16: 428-439, 2015.

10. Matuschak GM and Lechner AJ: Acute lung injury and the acute respiratory distress syndrome: Pathophysiology and treatment. Mo Med 107: 252-258, 2010.

11. Ding Q, Liu GQ, Zeng YY, Zhu JJ, Liu ZY, Zhang X and Huang JA: Role of IL-17 in LPS-induced acute lung injury: An in vivo study. Oncotarget 8: 93704-93711, 2017.

12. Wang T, Hou W and Fu Z: Preventative effect of OMZ-SPT on lipopolysaccharide-induced acute lung injury and inflammation via nuclear factor-kappa B signaling in mice. Biochem Biophys Res Commun 485: 284-289, 2017.

13. Cheng K, Yang A, Hu X, Zhu D and Liu K: Curcumin attenuates pulmonary inflammation in lipopolysaccharide induced acute lung injury in neonatal rat model by activating peroxisome proliferator-activated receptor $\gamma($ PPAR $\gamma)$ pathway. Med Sci Monit 24: 1178-1184, 2018.

14. Rigouzzo A, Khoy-Ear L, Laude D, Louvet N, Moutard ML, Sabourdin N and Constant I: EEG profiles during general anesthesia in children: A comparative study between sevoflurane and propofol. Paediatr Anaesth: Jan 4, 2019 (Epub ahead of print).

15. Vasileiou I, Xanthos T, Koudouna E, Perrea D, Klonaris C, Katsargyris A and Papadimitriou L: Propofol: A review of its non-anaesthetic effects. Eur J Pharmacol 605: 1-8, 2009.

16. Jiang S, Liu Y, Huang L, Zhang F and Kang R: Effects of propofol on cancer development and chemotherapy: Potential mechanisms. Eur J Pharmacol 831: 46-51, 2018.

17. Liu XR, Cao L, Li T, Chen LL, Yu YY, Huang WJ, Liu L and Tan XQ: Propofol attenuates $\mathrm{H}_{2} \mathrm{O}_{2}$-induced oxidative stress and apoptosis via the mitochondria- and ER-medicated pathways in neonatal rat cardiomyocytes. Apoptosis 22: 639-646, 2017.

18. Kaur J, Flores Gutiérrez J and Nistri A: Neuroprotective effect of propofol against excitotoxic injury to locomotor networks of the rat spinal cord in vitro. Eur J Neurosci 44: 2418-2430, 2016. 
19. Zheng X,Huang H,Liu J,Li M,Liu M and Luo T: Propofol attenuates inflammatory response in LPS-activated microglia by regulating the miR-155/SOCS1 pathway. Inflammation 41: 11-19, 2018.

20. Liu S, Sun JY, Ren LP, Chen K and Xu B: Propofol attenuates intermittent hypoxia induced up-regulation of proinflammatory cytokines in microglia through inhibiting the activation of NF-Bк/p38 MAPK signalling. Folia Neuropathol 55: 124-131, 2017.

21. Yuan D, Su G, Liu Y, Chi X, Feng J, Zhu Q, Cai J, Luo G and Hei Z: Propofol attenuated liver transplantation-induced acute lung injury via connexin43 gap junction inhibition. J Transl Med 14: 194, 2016.

22. Liu W, Zhu H and Fang H: Propofol potentiates sevoflurane-induced inhibition of nuclear factor- $\kappa \mathrm{B}$-mediated inflammatory responses and regulation of mitogen-activated protein kinases pathways via toll-like receptor 4 signaling in lipopolysaccharide-induced acute lung injury in mice. Am J Med Sci 354: 493-505, 2017.

23. Tan Z, Wang H, Sun J and Li M: Effects of propofol pretreatment on lung morphology and heme oxygenase-1 expression in oleic acid-induced acute lung injury in rats. Acta Cir Bras 33: 250-258, 2018.

24. Ma J, Xiao W, Wang J, Wu J, Ren J, Hou J, Gu J, Fan K and Yu B: Propofol inhibits NLRP3 inflammasome and attenuates blast-induced traumatic brain injury in rats. Inflammation 39 2094-2103, 2016.

25. Lv H, Zhu C, Liao Y, Gao Y, Lu G, Zhong W, Zheng Y, Chen W and $\mathrm{Ci} \mathrm{X}$ : Tenuigenin ameliorates acute lung injury by inhibiting NF- $\kappa \mathrm{B}$ and MAPK signalling pathways. Respir Physiol Neurobiol 216: 43-51, 2015.

26. Hu X, Shen H, Wang Y and Zhao M: Liver X receptor agonist TO901317 attenuates paraquat-induced acute lung injury through inhibition of $\mathrm{NF}-\kappa \mathrm{B}$ and JNK/p38 MAPK signal pathways. Biomed Res Int 2017: 4652695, 2017.

27. Grailer JJ, Canning BA, Kalbitz M, Haggadone MD, Dhond RM, Andjelkovic AV, Zetoune FS and Ward PA: Critical role for the NLRP3 inflammasome during acute lung injury. J Immunol 192: 5974-5983, 2014

28. Wang S, Zhao J, Wang H, Liang Y, Yang $\mathrm{N}$ and Huang $\mathrm{Y}$ : Blockage of P2X7 attenuates acute lung injury in mice by inhibiting NLRP3 inflammasome. Int Immunopharmacol 27: 38-45, 2015.

29. Bayne K: Revised guide for the care and use of laboratory animals available. American physiological society. Physiologist 39: 199, 208-211, 1996

30. Wang X, Liu C and Wang G: Propofol protects rats and human alveolar epithelial cells against lipopolysaccharide-induced acute lung injury via inhibiting HMGB1 expression. Inflammation 39: 1004-1016, 2016

31. Wang C, Zeng L, Zhang T, Liu J and Wang W: Casticin inhibits lipopolysaccharide-induced acute lung injury in mice. Eur J Pharmacol 789: 172-178, 2016.

32. Livak KJ and Schmittgen TD: Analysis of relative gene expression data using real-time quantitative PCR and the 2(-Delta Delta C(T)) method. Methods 25: 402-408, 2001

33. Gando S, Kameue T, Matsuda N, Sawamura A, Hayakawa M and Kato H: Systemic inflammation and disseminated intravascular coagulation in early stage of ALI and ARDS: role of neutrophil and endothelial activation. Inflammation 28: 237-244, 2004.
34. Azevedo ZM, Moore DB, Lima FC, Cardoso CC, Bougleux R, Matos GI, Luz RA, Xavier-Elsas P, Sampaio EP, Gaspar-Elsas MI and Moraes MO: Tumor necrosis factor (TNF) and lymphotoxin-alpha (LTA) single nucleotide polymorphisms: Importance in ARDS in septic pediatric critically ill patients. Hum Immunol 73: 661-667, 2012.

35. Rabelo MAE, Lucinda LMF, Reboredo MM, da Fonseca LMC, Reis FF, Fazza TF, Brega DR, de Paoli F, de Souza da Fonseca A and Pinheiro BV: Acute lung injury in response to intratracheal instillation of lipopolysaccharide in an animal model of emphysema induced by elastase. Inflammation 41: 174-182, 2018.

36. Chen C, Shi L, Li Y, Wang X and Yang S: Disease-specific dynamic biomarkers selected by integrating inflammatory mediators with clinical informatics in ARDS patients with severe pneumonia. Cell Biol Toxicol 32: 169-184, 2016.

37. Abderrazak A, Syrovets T, Couchie D, El Hadri K, Friguet B, Simmet $\mathrm{T}$ and Rouis M: NLRP3 inflammasome: From a danger signal sensor to a regulatory node of oxidative stress and inflammatory diseases. Redox Biol 4: 296-307, 2015.

38. Franco ML, Waszak P, Banalec G, Levame M, Lafuma C, Harf A and Delacourt C: LPS-induced lung injury in neonatal rats: Changes in gelatinase activities and consequences on lung growth. Am J Physiol Lung Cell Mol Physiol 282: L491-L500, 2002.

39. Miotla JM, Teixeira MM and Hellewell PG: Suppression of acute lung injury in mice by an inhibitor of phosphodiesterase type 4 Am J Respir Cell Mol Biol 18: 411-420, 1998.

40. Shi D, Zheng M, Wang Y, Liu C and Chen S: Protective effects and mechanisms of mogroside $\mathrm{V}$ on LPS-induced acute lung injury in mice. Pharm Biol 52: 729-734, 2014

41. Sun A, Wang W, Ye X, Wang Y, Yang X, Ye Z, Sun X and Zhang C: Protective effects of methane-rich saline on rats with lipopolysaccharide-induced acute lung injury. Oxid Med Cell Longev 2017: 7430193, 2017.

42. Wang J, Li R, Peng Z, Zhou W, Hu B, Rao X, Yang X and Li J: GTS-21 reduces inflammation in acute lung injury by regulating M1 polarization and function of alveolar macrophages. Shock: 30 Mar 2018 (Epub ahead of print).

43. Gao S, Li H, Zhou XQ, You JB, Tu DN, Xia G, Jiang JX and Xin C: Withaferin A attenuates lipopolysaccharide-induced acute lung injury in neonatal rats. Cell Mol Biol (Noisy-le-grand) 61: 102-106, 2015.

44. Hua S, Liu X, Lv S and Wang Z: Protective effects of cucurbitacin $\mathrm{B}$ on acute lung injury induced by sepsis in rats. Med Sci Monit 23: 1355-1362, 2017.

45. Dong Z and Yuan Y: Accelerated inflammation and oxidative stress induced by LPS in acute lung injury: Inhibition by ST1926. Int J Mol Med 41: 3405-3421, 2018.

46. Du L, Hu X, Chen C, Kuang T, Yin H and Wan L: Seabuckthorn paste protects lipopolysaccharide-induced acute lung injury in mice through attenuation of oxidative stress. Oxid Med Cell Longev 2017: 4130967, 2017.

47. Park HJ, Shin JY, Kim MH and Park BJ: Increased use in propofol and reported patterns of adverse events among anesthetics in Korea. Regul Toxicol Pharmacol 71: 478-483, 2015.

48. Bonnet U and Scherbaum N: Craving dominates propofol addiction of an affected physician. J Psychoactive Drugs 44: 186-190, 2012.

49. Bonnet U: Assessment of the addictive risk of propofol. Fortschr Neurol Psychiatr 79: 442-452, 2011 (In German). 